Journal of Attention Disorders Vol. 12(4):372-380 (2008)

ISSN: 1087-0547

doi:10.1177/1087054708314602

This is a peer reviewed pre-print version of the following article: Discriminating Between Children With ADHD and Classmates Using Peer Variables, which has been published in final form at:

http://www.sagepub.com/home.nav

http://jad.sagepub.com/

http://jad.sagepub.com/content/12/4/372.full.pdf+html

(C) 2008 Sage Publications Ltd.

\title{
Discriminating Between Children With ADHD and Classmates Using Peer Variables
}

\author{
Sylvie Mrug, University of Alabama at Birmingham, Betsy Hoza, University of \\ Vermont, Alyson C. Gerdes, Marquette University, Stephen Hinshaw, University of \\ California, Berkeley, L. Eugene Arnold, Ohio State University, Lily Hechtman, \\ McGill University, William E. Pelham, State University of New York at Buffalo
}

Objective:

Impaired peer relationships have long been recognized as one of the major functional problems of children with ADHD, but no specific guidelines on clinical levels of impairment in this domain exist.

Method:

This study used Receiver Operating Characteristics methodology to determine what aspects of peer functioning best discriminate between children with ADHD and their classmates. Optimal cutoffs indicative of clinical levels of impairment associated with ADHD diagnosis were determined for all variables. The participants were 165 children with AD/HD who were part of the Multimodal Treatment Study of Children With ADHD and their 1,298 classmates.

Results:

Variables that best discriminated between children with ADHD and their classmates included peer rejection and negative imbalance between given and received liking ratings (i.e., children with ADHD liked others more than they were liked).

Conclusion:

Peer rejection and negative imbalance show most promise for identifying clinically significant levels of peer relationship impairment in children with ADHD. (J. of Att. Dis. 2009; 12(4) 372-380).

ADHD is a prevalent psychological diagnosis in childhood, occurring in 3\% to $7 \%$ of school-age children (American Psychiatric Association, 2000). Although symptoms of hyperactivity, impulsivity, and inattention make up the core diagnostic criteria for ADHD, other impairing problems often co-occur with this disorder. These include oppositional behavior and conduct problems, academic failure, and difficulty in relationships with peers and adults (Hoza, Owens, \& Pelham, 1999). Given the importance of peer relationships for children's development (see Rubin, Bukowski, \& Parker, 1998), problems in peer relationships represent some of the most disconcerting functional problems that children with ADHD experience (Whalen \& Henker, 1992). Studies that compare the social status of these children with non-ADHD peers report unanimously that ADHD children are more often rejected by the peer group (Hinshaw \& Melnick, 1995; Hodgens, Cole, \& Boldizar, 2000; see Mrug, Hoza, \& Gerdes, 2001, for a review), but their peer difficulties involve other aspects of peer relationships as well (e.g., lack of friendships or imbalance between liking others and being liked by others; Hoza et al., 2005). Impairment in peer and social functioning affects both boys and girls with ADHD (Greene et al., 
2001; Hinshaw, 2002). Children with ADHD become rejected within hours or days of entering a new peer group (Erhardt \& Hinshaw, 1994; Pelham \& Bender, 1982), primarily due to their negative, disruptive behavior and lack of social skills (Mrug, Hoza, Pelham, Gnagy, \& Greiner, 2007; Whalen \& Henker, 1992). Difficulties in peer relationships in these children often persist into adolescence (Bagwell, Molina, Pelham, \& Hoza, 2001), and their impairment in social functioning predicts negative outcomes later on, including conduct disorder and substance use problems (Greene, Biederman, Faraone, Sienna, \& Garcia-Jetton, 1997).

Despite peer problems representing a key domain of impairment that needs to be considered in the assessment process, there are no guidelines on what represents enough impairment in peer relationships to be suggestive of a diagnosis of ADHD. Traditionally, research on the assessment of ADHD has focused largely on the core symptoms of inattention and hyperactivity/impulsivity rather than functional impairment (e.g., Frick et al., 1994). However, as Pelham, Fabiano, and Massetti (2005) point out, impairment in functioning and not the core ADHD symptoms is typically the main reason for referral. Also, specific impairments, in particular problems in peer relationships, are better predictors of long-term outcomes than the core symptoms of ADHD (see Pelham et al., 2005). Developing decision guidelines for clinical levels of peer relationship problems in children with ADHD would thus be of great importance for clinicians and researchers alike. By improving clinicians' ability to identify children with significant peer problems, it would help in the diagnostic process, suggest peer-related treatment goals, and aid in the evaluation of treatment effectiveness. In addition, such guidelines would enable researchers to select children with both ADHD and substantial peer problems for descriptive and intervention studies. Moreover, specific levels of impairments in peer relationships that are best predictive of ADHD may generalize to other clinical disorders that have a negative effect on peer functioning (e.g., pervasive developmental disorders). Previous studies used arbitrary cutpoints to classify children into impaired versus not impaired categories (e.g., one standard deviation above the mean on a measure of peer rejection; Coie, Dodge, \& Coppotelli, 1982). This is the first investigation that provides empirically derived criteria for levels of peer relationship impairment that are best predictive of ADHD diagnosis.

Specifically, we use the Receiver Operating Characteristics (ROC; Kraemer et al., 1997) methodology to determine optimal cutpoints on multiple peer variables that best distinguish between children with the combined subtype of ADHD and their classmates. We build directly on the results reported by Hoza et al. (2005), who completed the most comprehensive analysis of peer difficulties in children with ADHD to date. Hoza et al. analyzed differences between participants in the Multimodal Treatment Study of Children With ADHD (MTA; MTA Cooperative Group, 1999) and randomly selected classmates on a number of indices of peer functioning. All peer variables were derived from classwide sociometric procedures, a gold standard of assessing peer relationships in school-aged children. Hoza et al. found that, compared with their classmates, children with ADHD had lower social preference scores and higher social impact scores, were more disliked, had fewer dyadic friends, and received more negative and fewer positive ratings of liking than they gave to peers. In addition, children who nominated children with ADHD as somebody they "do not want to be friends with" had higher social preference scores and were better liked than children who gave negative nominations to nonADHD classmates.

In this investigation, we include the same peer variables used in Hoza et al. (2005), with two exceptions. We do not include variables on which there were no significant differences between children with ADHD and classmates because those variables clearly do not discriminate 
between the two groups. Also, we decided to use measures of peer acceptance and peer rejection instead of social preference and social impact for several reasons. (Measures of peer acceptance and rejection are based on the number of received positive and negative nominations, respectively. Social preference is then derived as the difference between peer acceptance and peer rejection, and social impact is computed as the sum of peer acceptance and peer rejection scores.) First, because social impact and social preference can only be computed if measures of both peer acceptance and rejection are available, providing results for these primary constructs may be more useful for future studies that are not able to gather both (e.g., school administrators are often reluctant to allow researchers to measure peer rejection). Second, the use of acceptance and rejection is more in line with past studies of the peer relationships of children with ADHD, which relied almost exclusively on these constructs. In contrast, social impact and preference are commonly used in the developmental literature on peer relationships. Finally, social preference is typically highly correlated with both acceptance and rejection, so similar results would be obtained with either preference or acceptance and rejection.

In summary, the main goal of this study is to derive empirically supported cutpoints on multiple measures of peer functioning that would provide optimal discrimination between children with ADHD and their classmates. Specifically, the best cutoffs will be chosen to maximize overall accuracy by minimizing both false positive and false negative classification of ADHD status.

\section{Method}

\section{Participants}

The participants in the study came from 165 classrooms, with each classroom containing 1 child with ADHD and 5 to 16 participating same-sex classmates. It is customary to solicit only same-sex nominations in school-aged children, because as many as $90 \%$ of children's friendships in this age group involve same-sex peers (Kupersmidt, DeRosier, \& Patterson, 1995). Hence, data were obtained for 130 boys with ADHD and their 1,026 male classmates, and 35 girls with ADHD and their 272 female classmates. The ADHD participants were recruited as part of the MTA (MTA Cooperative Group, 1999) at three of the six study sites that chose to administer sociometric assessments at baseline, before any treatment began. The MTA participants were 7.0 to 9.9 years old at the time of study entry. The mean, median, and mode of the number of participating children in each classroom were 8.9, 8.0, and 8.0, respectively. For more information about the sample, please refer to Hoza et al. (2005).

\section{Procedure}

Only children whose parents gave informed consent participated in the study. The mean participation rate in the 165 classrooms was $64 \%$, which results in relatively accurate sociometric data (Crick \& Ladd, 1989). The assessments were conducted in individual sessions with all first and second graders and with any classrooms of older children whose teacher suspected that three or more participants in that class would have difficulty reading the forms. In all other classrooms, the assessments were completed in a group format with the instructions read aloud and individual help given to any children who needed it.

Three types of sociometric measures were collected: positive nominations, negative nominations, and ratings of liking. For each measure, children who received a group administration were given a list of all participating samesex classmates. For positive nominations, children were asked to circle the names of all children who were their "best friends 
in the class." Next, they were asked to identify up to three of the selected classmates as their very best friends and number them in the order of their first best friend, second best friend, and third best friend. For negative nominations, children were asked to circle the names of all classmates on the list that they "DO NOT want to be friends with." They were not asked to number their negative choices for ethical reasons. For children who received individual administrations, the procedure was similar with the exception that individual name cards were used instead of lists (for both positive and negative nominations) and children made their selections by picking up or pointing to the cards. Finally, children provided ratings of liking for every participating classmate using a 5-point scale ranging from really like (1) to really do not like (5). "Smiley" and "frowney" faces were used to anchor the positive and negative ends of the scale, respectively. Younger children receiving individual administrations were presented with individual name cards, one at a time, and all points on the rating scale were accompanied by explanatory faces.

\section{Variables Derived From the Sociometric Data}

\section{Peer acceptance.}

The number of positive nominations (including all nominations, not just the top three best friends) each child received from his or her classmates was tallied and standardized within each class to adjust for differential participation rates across classrooms. The peer acceptance score thus indicates the extent to which the same-sex peer group accepts the child.

Peer rejection.

Similarly, the number of negative nominations received from the child's classmates was tallied and standardized within class. Peer rejection indicates the extent to which each child is disliked or rejected by the same-sex peer group.

Average liking.

An average was computed from the liking ratings received from all same-sex classmates. Consistent with the individual ratings, average scores can range from 1 (child is really liked) to 5 (child is really not liked).

\section{Positive and negative imbalance.}

These scores indicate a positive and negative difference between ratings of liking that the child gave to others and those that he or she received from others. Positive imbalance was computed as the sum of all absolute differences between the child's given and received scores when the child received a more positive liking rating than he or she gave to others. Conversely, negative imbalance was computed as the sum of the absolute differences when the child received a more negative rating that he or she gave. Both imbalance scores were standardized within class to adjust for different numbers of participants across classes. High negative imbalance thus indicates that the child received more negative liking scores than he or she gave, whereas high positive imbalance scores indicate that the child received more positive ratings than he or she gave.

\section{Dyadic friendship.}

The number of dyadic friendships was computed for each child. To be counted as a dyadic friendship, both the child and the classmate must have selected each other on the positive nomination procedure. To achieve greater variability and more possible cutpoints on this variable, we based dyadic friendship on unlimited positive nominations, although this represents 
a deviation from our prior work (Hoza et al., 2005), which used only first and second choice nominations. In other words, any classmate circled by a child on the positive nominations could be a dyadic friend as long as he or she also circled the target child in return.

Preference and liking of negatively nominating peers.

Based on Hoza et al. (2005), children's individual preference and liking scores were averaged (separately for preference and liking) across all classmates who selected the target child on the negative nomination procedure. We only included average liking and social preference scores of negatively nominating peers because other variables did not differ between children with ADHD and their classmates in prior work (Hoza et al., 2005); hence, they would be expected to show poor discrimination between the groups.

\section{Results}

This study used the ROC approach to compare the utility of various peer variables in discriminating between children with ADHD and their classmates and to derive optimal classification cutpoints. Using ROC software designed by Kraemer (1992), we first computed sensitivity and specificity values for all cutpoints within the range of each variable (in increments of .10). The sensitivity is computed as the probability that a child with ADHD will be classified in the ADHD group (i.e., how sensitive the test is to detect the disorder), whereas specificity is the probability that a classmate will be classified as not- ADHD (i.e., whether the test is specific just to children with ADHD). To increase the reliability of results, only cutoffs that had a marginal count of at least 10 cases above and below the cutoff were used. The sensitivity and specificity values for these cutpoints were then plotted against each other to create the ROC curves that are presented in Figure 1. The larger the area under the curve, or equivalently, the closer the curve is to the right upper corner of the graph, the better the variable discriminates between ADHD participants and their classmates. It can be seen in the figure that the best discriminating variables were peer rejection, average liking, and negative imbalance, followed by peer acceptance and positive imbalance, in this order. Number of dyadic friends and preference and liking of negatively nominating peers, on the other hand, did not discriminate between the two groups nearly as well.

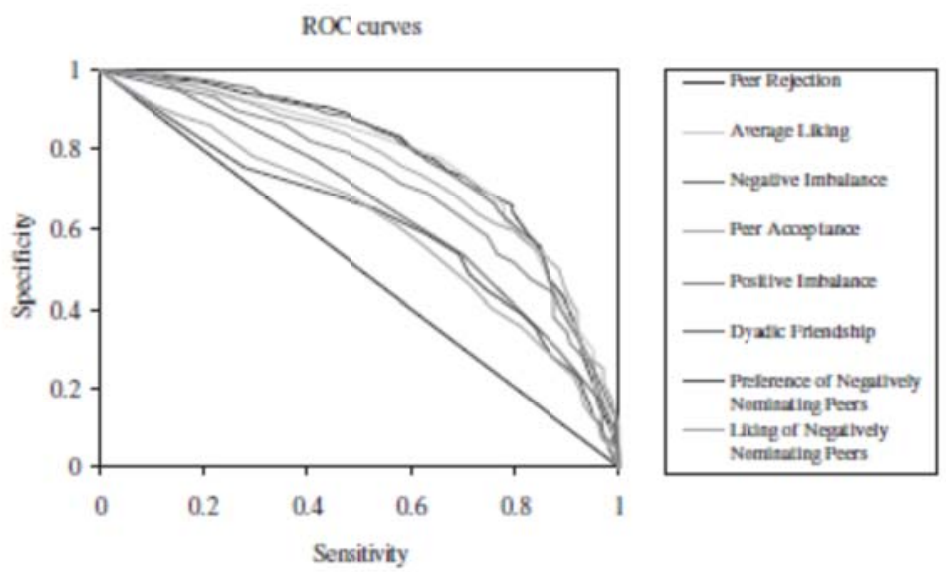

Note: Color figure is available at http://jad.sagepub.com

Figure 1 Receiver Operating Characteristics (ROC) Curves for all Peer Variables 